\title{
iCore: A Cognitive Management Framework for the Internet of Things
}

\author{
Raffaele Giaffreda \\ CREATE-NET, Trento, Italy \\ raffaele.giaffreda@create-net.org
}

\begin{abstract}
Core is an EU FP7 Integrated Project aimed at leveraging on the use of cognitive technologies for empowering the Internet of Things to deliver on the current expectations which see it as one of the main pillars of the Future Internet. The project brings together a strong set of industrial Partners, mostly from Europe but spanning also China and Japan which collaborate with research centers and universities to deliver solutions that address heterogeneity and reusability of IoT objects while striving for self-management capabilities that keep low complexity as the numbers of interconnected objects increase exponentially.
\end{abstract}

\section{$1 \quad$ Key Issues and Objectives}

iCore is an EU FP7 project in the Internet of Things (IoT) domain. The project aims to address some of the numerous challenges posed by the "7 trillion devices for 7 billion people" paradigm, namely the technological heterogeneity that derives from such vast amounts of heterogeneous interconnected objects and the complexity this future vision entails.

In order to achieve these objectives iCore proposes a cognitive management framework for the Internet of things which on the one hand investigates the means to virtualise interconnected objects, while on the other aims to leverage on the use of cognitive technologies to achieve self-management of these objects while considering the needs and requirements of the application / user to be supported.

iCore solutions will contribute to the ability of the Internet of Things to achieve its full potential providing architectural and technology foundations that foster the easy creation of innovative applications and exploit the increasingly widespread fabric of sensing and actuating IoT objects.

Obvious beneficiaries of such innovation, besides end-users, are expected to be manufacturers of objects that thanks to solved interoperability issues can promote reuse of their devices in many different application contexts. iCore solutions will also open-up new opportunities for all stakeholders traditionally associated with smartcities scenarios (energy, water, traffic, waste etc.), which will have to face the challenge of homogeneous interactions with and the ability to extract relevant and compartmentalised knowledge from ubiquitous sensing capabilities of IoT. 


\section{Technical Approach}

To achieve these objectives iCore proposes a cognitive framework comprising three levels of functionality, exposing well defined interfaces which make the functionality therein reusable for various and diverse applications.
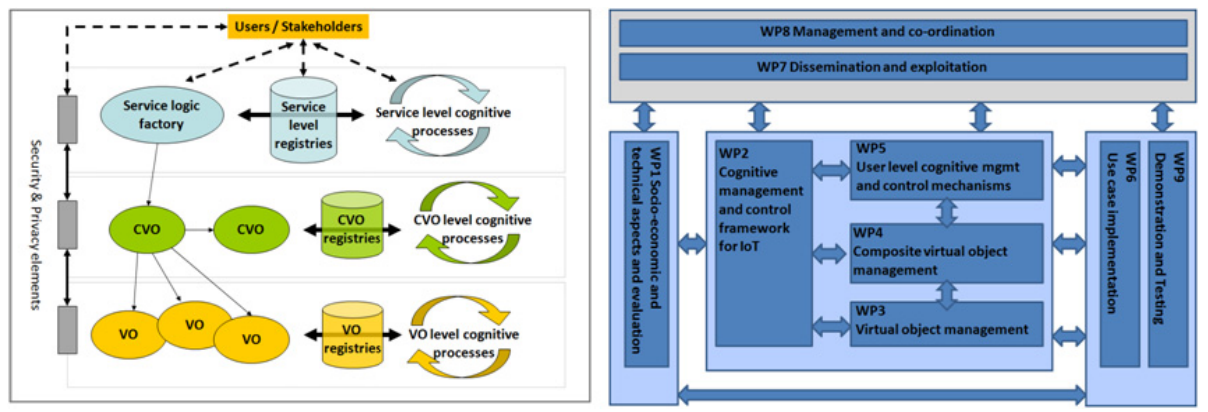

Fig. 1. iCore Cognitive Framework and work-package structure

Starting from the bottom of Fig. 1, the first level is the Virtual Object (VO) level, dedicated to the characterisation of the VO abstraction, meant to address interoperability and heterogeneity issues of IoT. Part of this abstraction also foresees the semantic enrichment of the description of Real World Objects, according to an RDF model, therefore contributing to the issue of automatic re-use of objects in diverse application contexts.

The next level up is dedicated to the set of functional blocks that realise the Composite Virtual Object (CVO) concept, whereby individual VOs are mashed-up to provide more composite functionality that dynamically binds the requirements of the Service Level to the continuously changing availability of underlying IoT resources. In this way the CVO level contributes to an iCore platform the ability to maintain "system knowledge".

The top level, called Service Level, is the one that exposes interfaces towards applications or users, as it's meant to translate Service Requests from outside the iCore platform into actions that, starting from service templates dynamically deploy, bind and run the set of processes that satisfy the request. This level is also responsible for maintaining and growing so called "real world knowledge" within the platform, to ensure that the knowledge supporting iCore autonomic decisions is indeed representative of the actual and changing application / user situation contextual background.

These three levels are developed in three separate work-packages, while the crosscutting concerns at architectural, cognitive management and security \& privacy and level are factored out in a separate technical coordination work-package.

The proposed solutions are to be validated through three further work-packages, one dedicated to socio-economic and business aspects, one to the implementation of four use cases (ambient assisted living, smart office, smart transportation, and supply chain management) and one dedicated to the implementation of demonstration trials. 


\section{iCore profile}

The iCore Consortium consists of 21 partners with a strong industrial component represented by 8 ICT manufacturers, telecom operators, software vendors, system integrators,

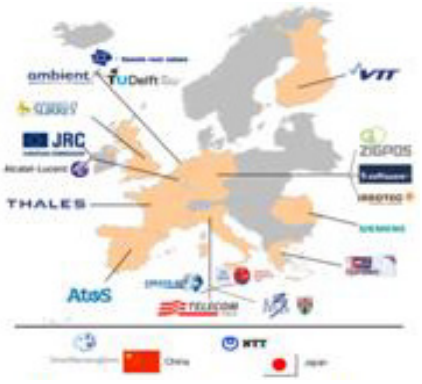

Fig. 2. iCore Consortium software service/end users (Siemens, Thales, Alcatel-Lucent, Fiat, Atos, Software AG, NTT and Telecom Italia). 5 SMEs are part of the consortium (Ambient systems, ZIGPOS, Innotec21, SSS and M3S) as well as 4 internationally recognised research centres (Create-net, EC JRC, TNO, VTT) and 4 universities (University of Surrey, University of Piraeus, Technical University of Delft and University of Genova). Overall iCore size is $8.7 \mathrm{mEur}$ of EU funding and the project is expected to last 3 years (it started in October 2011).

\section{Expected Impact}

iCore solutions enable a variety of stakeholders to take part in the easy creation and delivery of value-add IoT services which can be dynamically tailored to the users situation and needs.

The iCore technology is designed to be managed and used by multiple stakeholders, driven by different business models and various interests, from platform operators to device manufacturers, to SMEs concentrating on bespoke services around their core IoT business. iCore will allow an adequate level of interoperability, so that innovative and competitive cross-domain systems and applications can be developed. In fact infrastructure and concepts are aimed at being agnostic of the application domain in which they will be used and this is where the strength of its solutions lies from an impact perspective.

The interconnection of physical objects and VOs in iCore will amplify the profound effects that large-scale networked communications are having on our society, gradually resulting in a genuine paradigm shift. Future Internet and IoT are becoming more and more a vital resource to economy and society, as vital processes in society, be it energy distribution, traffic management and (food) logistics are being coordinated by and thus heavily depend on means of telecommunication networks.

Open Access. This article is distributed under the terms of the Creative Commons Attribution Noncommercial License which permits any noncommercial use, distribution, and reproduction in any medium, provided the original author(s) and source are credited.

\section{Reference}

1. iCoreProject WebSite, http: //www. iot-icore.eu 